\title{
Acute Kidney Injury in Asia
}

\author{
Li Yang \\ Renal Division, Department of Medicine, Peking University First Hospital, Peking University Institute of \\ Nephrology, Key Laboratory of Renal Disease, Ministry of Health of China, and Key Laboratory of \\ Chronic Kidney Disease Prevention and Treatment, Peking University, Ministry of Education, Beijing, China
}

\section{Key Words}

Acute kidney injury · Cause · Epidemiology · Recognition ·

Renal replacement therapy

\begin{abstract}
Background: Acute kidney injury (AKI) is a common disorder and is associated with a high morbidity and mortality worldwide. The diversity of the climate and of the socioeconomic and developmental status in Asia has a great influence on the etiology and presentation of $\mathrm{AKI}$ in different regions. In view of the International Society of Nephrology's 0 by 25 initiative, more and more attention has been paid to $\mathrm{AKI}$ in Asian countries. Summary: In this review, we summarize the recent achievements with regard to the prevalence and clinical patterns of AKI in Asian countries. Epidemiological studies have revealed the huge medical and economic burden of AKI in Eastern Asian countries, whereas the true epidemiological picture of AKI in the tropical areas is still not well understood. In high-income Asian regions, the presentation of AKI resembles that in other developed countries in Europe and North America. In low-income regions and tropical areas, infections, environmental toxins, and obstetric complications remain the major culprits in most cases of AKI. Preventive opportunities are missed because of failure to recognize the risk factors and early signs of AKI. Patients often present late for treatment or are recognized late by physi-
\end{abstract}

cians, which leads to more severe kidney injury, multiorgan involvement, and increased mortality. There is significant undertreatment of $\mathrm{AKI}$ in many regions, and medical resources for renal replacement therapy are not universally available. Key Messages: More efforts should be made to increase public awareness, establish preventive approaches in communities, educate health-care practitioner entities to achieve better recognition, and form specialist renal teams to improve the treatment of AKI. The choice of renal replacement therapy should fit patients' needs, and peritoneal dialysis can be practiced more frequently in the treatment of AKI patients. Facts from East and West: (1) More than $90 \%$ of the patients recruited in AKI studies using KDIGO-equivalent criteria originate from North America, Europe, or Oceania, although these regions represent less than a fifth of the global population. However, the pooled incidence of AKI in hospitalized patients reaches $20 \%$ globally with moderate variance between regions. (2) The lower incidence rates observed in Asian countries (except Japan) may be due to a poorer recognition rate, for instance because of less systematically performed serum creatinine tests. (3) AKI patients in South and Southeastern Asia are younger than in East Asia and Western countries and present with fewer comorbidi-

For acute kidney injury in Western countries, see Bouchard and Mehta, Kidney Dis 2016;2:103-110.

\section{KARGER}

E-Mail karger@karger.com

www.karger.com/kdd
(C) 2016 S. Karger AG, Basel

2296-9381/16/0023-0095\$39.50/0
Li Yang, MD

Renal Division, Department of Medicine

Peking University First Hospital

No. 8 Xi Shi Ku Street, Beijing 100034 (China)

E-Mail li.yang@bjmu.edu.cn 
ties. (4) Asian countries (and to a certain extent Latin America) face specific challenges that lead to AKI: nephrotoxicity of traditional herbal and less strictly regulated nonprescription medicines, environmental toxins (snake, bee, and wasp venoms), and tropical infectious diseases (malaria and leptospirosis). A higher incidence and less efficient management of natural disasters (particularly earthquakes) are also causes of AKI that Western countries are less likely to encounter. (5) The incidence of obstetric AKI decreased globally together with an improvement in socioeconomic levels particularly in China and India in the last decades. However, antenatal care and abortion management must be improved to reduce AKI in women, particularly in rural areas. (6) Earlier nephrology referral and better access to peritoneal dialysis should improve the outcome of AKI patients.

(c) 2016 S. Karger AG, Basel

\section{Introduction}

Acute kidney injury (AKI) is a common disorder and is associated with a high morbidity and mortality worldwide. In 2013, the International Society of Nephrology (ISN) put forth the human rights case statement of 0 by 25 [1], i.e. no one should be dying of untreated AKI in lowresource regions by 2025, with an emphasis on resourcepoor countries in Africa, Latin America, and Asia with disadvantaged populations and poor access to medical care.

Asia is the Earth's largest and most populous continent, and there is great variation across and within regions with regard to climate, geographic features, ethnic groups, cultures, socioeconomic status, and developmental heterogeneity. Climates range from arctic and subarctic in Siberia to tropical in South and Southeast Asia. This diversity has a great influence on the prevalence, etiology, and clinical features of AKI in different regions. The disease pattern in South and Southeast Asia shows tropical features; in some high-income Eastern Asian regions, the disease pattern resembles that of other developed countries in Europe and North America, whereas in the northern, middle, western, and temperate regions of Eastern Asia, the disease patterns are different. As in all world regions, the wealth of Asia differs widely between and within states. Except for Japan, Korea, Taiwan, Singapore, and Hong Kong, care for AKI is not universally available to all patients [2]. During the 20th century, Asia has exhibited economic dynamism as well as a robust population growth, which led to great changes in the living environments, eating habits, disease spectrum, and medical care conditions. In view of the ISN 0 by 25 initiative [1], more and more attention has been paid to AKI in Asia. The present review focuses on the epidemiology, patterns, and current situation of recognition and treatment of AKI in Asia.

\section{Epidemiology of AKI in Asia}

In recently published meta-analyses of the global burden of AKI [2,3], the pooled incidence of AKI in the hospitalized population studied according to KDIGO-equivalent criteria was 19.4\% in Eastern Asia, 7.5\% in Southern Asia, 31.0\% in Southeastern Asia, 9.0\% in Central Asia, and $16.7 \%$ in Western Asia. The pooled mortality of AKI patients was 36.9\% in Eastern Asia, 13.8\% in Southern Asia, and 23.6\% in Western Asia [3]. These high incidence rates of $\mathrm{AKI}$ in hospitalized patients reveal a huge medical burden of AKI in Asia, as in all world regions. However, it is still difficult to estimate the exact prevalence of AKI in Asia due to the limited number of studies that could be included in the meta-analysis. Firstly, all the publications originate from large academic hospitals, and most of them focused on a special patient population with a high risk of AKI, such as patients in emergency or critical care units as well as patients undergoing cardiac surgery, exposed to nephrotoxins, with sepsis, and after trauma [3]. This population selection bias would lead to an overestimation of the burden of hospital-acquired AKI if these data were used as representative of AKI in the general population in different regions or countries. On the other hand, underreporting of AKI has been well acknowledged in low-income regions with a lack of serum creatinine tests and a low awareness of AKI by health practitioners. Besides, there are almost no data on the incidence of AKI in rural areas. Therefore, the prevalence of AKI in low-income regions has been considerably underestimated, and the magnitude of community-acquired $\mathrm{AKI}$ (CA-AKI) is virtually unknown.

\section{General Hospital Settings}

Recently, the ISN 0by25 China consortium launched a nationwide survey on AKI in mainland China [4] which screened 2.2 million hospitalized adult patients in 44 academic/local general hospitals across the country. The detection rate of AKI was $1 \%$ according to KDIGO criteria and $2 \%$ according to expanded criteria ( $\geq 50 \%$ change during hospital stay) with an in-hospital mortality rate of $12.4 \%$. Based on this survey, it is estimated that nearly 3 million AKI patients have been treated in hospitals in 
China during 2013, among which 700,000 patients died. This study, even though a substantial medical burden from AKI in China was shown, still vastly underestimates the prevalence of AKI, since only $25 \%$ of the hospitalized patients had repeated serum creatinine assays. In another recently published multicenter survey on AKI from 9 tertiary academic hospitals located in 7 big cities of mainland China [5], the detection rate of AKI (according to KDIGO criteria) was about $2.3 \%$ in adult patients hospitalized in general hospitals ( $30 \%$ of the total of hospitalized patients having repeated serum creatinine assays) with $8.8 \%$ in-hospital deaths. After adjusting for the frequency of serum creatinine tests and risk factors for AKI, the authors reported an AKI incidence of $11.6 \%$ among 659,945 hospitalizations. This novel method potentially overcomes the insufficiency of serum creatinine assays in the studied population, whereas an overestimation of the AKI incidence might exist due to using the patients with daily serum creatinine data within a 7-day window as a reference to obtain the empirical relationship between detection rate and serum creatinine frequency, since these patients probably had a much higher risk for AKI and, thus, triggered more frequent monitoring of renal function during the hospital stay. Single-center studies from general tertiary hospitals of other Asian countries using KDIGO-equivalent criteria have shown an AKI incidence of $6.9 \%$ in Japan (69\% of hospitalized patients having repeated serum creatinine assays) [6], 1.2\% in Korea [7], and $0.9 \%$ in Turkey [8], with mortality rates of $17.5-34.6 \%$. The incidence rates reported in patients hospitalized in general hospitals from Asian countries, except for Japan, are much lower than those reported from Western countries (7-18\%) [9-11], where repeated serum creatinine tests are more common (63.2-67.6\%) [12, 13]. This suggests a significant underreporting of AKI in most parts of Asia due to insufficient monitoring of renal function in hospitalized patients.

\section{Intensive Care Unit Settings}

$\mathrm{AKI}$ is a frequent complication in the intensive care unit (ICU). According to the nationwide multicenter survey on AKI in China, around $30 \%$ of AKI cases are treated in the ICU [4]. The incidence of AKI is $30-50 \%$ in total ICU admissions, with $30 \%$ of the AKI cases reaching stage 3 and $27 \%$ dying during the hospital stay $[14,15]$. Similar rates were reported from single-center studies in Korea and Japan, with an AKI incidence of around $40 \%$ and in-hospital death in $26 \%[16,17]$. Higher incidence rates of AKI were reported from single ICU centers in Sri Lanka and Malaysia (60-65\%), with a higher percentage of patients reaching AKI stage 3 (56-58.5\%) and a higher rate of in-hospital mortality (30-52\%) [18]. The differences in incidence, severity, and mortality in ICU AKI settings between studies could be due to the variety in severe comorbidities, especially sepsis, whereas deaths that occur due to a delayed recognition of AKI or inadequate response to the diagnosis of AKI have not been discussed.

\section{Causes and Patterns of AKI in Asia}

Patterns of AKI are different across Asia. Patients from most regions of Eastern Asia, including China, Japan, Korea, and Taiwan, are older (average age 60-70 years) than those from Southern and Southeastern Asia [19], including India, Malaysia, Thailand, and Siri Lanka (average age $44-53$ years). A male predominance is reported in most studies from different regions (55-75\%). Chronic comorbidities, such as diabetes mellitus (19$28 \%)$, cardiovascular disease (35-41\%), chronic kidney disease (24-31\%), and malignancy (12-19\%), are more common in patients from Eastern Asia than in those from other Asian regions.

The causes of hospital-acquired AKI are similar in different regions, with renal hypoperfusion and nephrotoxic drugs being the most common insults. CA-AKI contributes more to the AKI entities in Southern and Southeastern Asia (76-84\%) $[19,20]$ than in Eastern Asia (30-54\%), with distinct disease patterns across the regions. Studies from tertiary hospitals in China showed that nephrotoxic drugs (30-39\%) and urinary tract obstruction (12\%) remain the most common causes of CAAKI [5]. In India [21], patients who develop CA-AKI are much younger (average age 40 years) than Chinese patients (58-64 years) and more frequently affected by infectious diseases (47\%), including malaria in $17 \%$, infectious diarrhea in $19 \%$, and sepsis in $11 \%$. Other common causes of CA-AKI in India include obstetric complications, animal and plant toxins, and the use of natural medicines.

\section{Drug-Induced AKI}

The kidneys are frequently exposed to drugs and/or toxic metabolites and are, therefore, a common site of drug toxicity. Various drugs can affect different compartments of the kidney, causing AKI through mechanisms including renal hypoperfusion, toxic and/or ischemic acute tubular necrosis, allergic acute interstitial nephritis, endothelial injury, autoimmune glomerulonephritis, and crystal intrarenal obstruction. Globally, drug nephrotox- 
icity contributes to $17-26 \%$ of AKI [22, 23]; however, distinct sociocultural behavior in the administration of medicine across Asian countries makes drug-induced AKI an important entity. Firstly, the supervision of pharmacology is less strictly managed in many Asian countries, which provides residents with easy access to a wide scope of nonprescription medications. Secondly, traditional herbal medicine is prevalent in most regions of Asia, and natural medicines form special classes of nephrotoxins encountered in traditional societies. These agents are usually prescribed by practitioners of traditional medicine in rural areas, especially in the regions with a lack of medical facilities. In China, around $40 \%$ of AKI is due to drug toxicity [5]. Nearly $70 \%$ of the AKI patients had had nephrotoxic medication exposure before or at the time when AKI developed [4], which indicates a negligible role of drug toxicity in triggering and promoting kidney injury in the majority of AKI patients.

Of the diverse medicines that can cause AKI, antimicrobial agents, nonsteroidal anti-inflammatory drugs, and herbal medicine are the most common types of nephrotoxic drugs in China $[4,5]$. In rural areas and tropical regions, herbal medicine plays a predominant role in causing drug-toxic AKI. Great efforts need to be made to increase the awareness of drug nephrotoxicity in the communities, to identify the common culprit drugs in the different regions, and to strengthen the supervision system for drug use. In most situations, prompt withdrawal of the offending agent generally results in renal recovery. However, if the culprit drugs are lifesaving or even irreplaceable, discontinuation of the medicine will strongly limit treatment. Thus, preventive approaches to counteract drug nephrotoxicity should be considered before the beginning of AKI development, which includes maintaining optimal renal perfusion, prescribing proper dosage and medication combinations, etc.

\section{Tropical Infectious Disease-Related AKI}

Malaria is a common infectious disease causing AKI in tropical communities of Asia [24], especially in regions of Southern India, Sri Lanka, Malaysia, Indonesia, Thailand, and Yunnan and Hainan provinces in South China. The disease is caused by the protozoan Plasmodium and transmitted by the Anopheles mosquito. Most malarial AKI occurs in connection with $P$. falciparum infection, with a few reported cases in the context of $P$. vivax infection. In addition to being a major medical burden to the native communities, malaria threatens nonimmune travelers and induces more severe conditions. The overall incidence of AKI is approximately $1-4 \%$ in P. falciparum ma- laria, whereas the rate increases to $20-60 \%$ in those with severe malaria disease $[25,26]$ and to $25-30 \%$ in nonimmune individuals [27]. About $15-80 \%$ of malarial AKI patients have been reported to require dialysis $[26,28]$, and the mortality rate varies between different studies (6$50 \%)[26,27]$. Prerenal AKI and acute tubular necrosis are the main patterns. Early antimalarial treatment, timely recognition of AKI, and initiation of dialysis when needed are associated with improved survival and recovery of renal function [29].

Leptospirosis is the most widespread zoonosis across the world, caused by Leptospira interrogans, and particularly affects the tropical regions including India, Indonesia, Cambodia, Thailand, Vietnam, the Philippines, and parts of China. The major animal hosts include rodents, dogs, cattle, sheep, pigs, rabbits, etc. Humans get the infection either by exposure to water contaminated with infected animal urine or occupational contact with the tissue of infected animals. Leptospira directly invades the kidney, causing injury through direct toxicity to the renal tubules and inducing a local inflammatory reaction. Approximately $10-85 \%$ of infected patients develop AKI $[30,31] ; 30 \%$ of the AKI cases require dialysis [32], and the mortality rate varies between different series (4-20\%) $[33,34]$. Patients with leptospirosis usually require supportive measures such as correction of electrolyte disorders, adequate fluid replacement, and dialysis in oliguric patients when needed. Although leptospirosis is usually self-limiting, early use of penicillin or doxycycline can shorten the duration of fever, alleviate disease severity, and hasten recovery.

Hemorrhagic fever with renal syndrome is a serious rodent-borne disease which occurs globally, but the majority of cases are located in Asian countries including China, Korea, and the far-eastern region of Russia. In China, there are 20,000-50,000 cases reported annually [35]. The etiologic agents are of the genus hantavirus, and the main natural reservoir hosts are the brown Norway rat and striped field mouse, which can be found in nearly all provinces in China. Clinical symptoms include fever, headache, back pain, abdominal pain, hypotension, multisystemic hemorrhage, and AKI [36, 37]. Prominent renal dysfunction is seen in $80-90 \%$ of the infected patients, and about $1 / 3$ of the cases develop severe AKI (stage 3 ) or need dialysis [37, 38]. Microvascular inflammation and acute tubular necrosis are the major histologic features of hantavirus-induced AKI [39]. The overall mortality varies from 0.5 to $12 \%$ according to different viral species, individual sensitivity, and the timing of diagnosis and treatment [38]. 
Environmental Nephrotoxin Exposure-Induced AKI

Environmental nephrotoxins including animals, insects, plants, and chemicals constitute important insults for CA-AKI in tropical regions of Asia. In China, environmental nephrotoxins contribute to $3-4 \%$ of CA-AKI entities and are more frequent in southern regions at a northern latitude of $20-30^{\circ}$ [4]. In Southern Asia and Southeastern Asia, the rate may be up to 10\% [40].

Poisonous snakebite-induced AKI contributes to 70\% of CA-AKI in Myanmar, 2-3\% in India, and 1-2\% in Thailand [24]. The mechanisms have been attributed to direct nephrotoxicity, hypovolemia, hemoglobinuria, myoglobinuria, and disseminated intravascular coagulation. Data from retrospective series reported an AKI incidence of $1.4-38 \%$ with a mortality rate ranging from 1 to $20 \%$ [41, 42] depending on the species. Kidney injury usually occurs within hours but can also occur as late as several days after the bite. More than $90 \%$ of the patients develop oliguria and require dialysis [42]. The most common pattern of $\mathrm{AKI}$ is acute tubular necrosis, followed by acute interstitial nephritis and acute renal cortical necrosis. Occasionally, necrotizing or crescentic glomerulonephritis can be seen. Early administration of specific monovalent antivenom, before AKI starts, is critical. Plasmapheresis can be the alternative treatment if antivenom is unavailable. Dialysis should be performed early and frequently.

Bee and wasp stings are common insults for CA-AKI in Asia, especially in regions of Southern China, India, Nepal, and Vietnam [43-46]. One or two stings cause just a local allergic reaction, but massive attacks with hundreds to thousands of stings cause severe systemic symptoms, affecting different organs with a high mortality. Patients with AKI have been reported to have received 22 to more than 1,000 stings. AKI develops directly from the nephrotoxic effect of the venoms and secondary to hemolysis and/or rhabdomyolysis, and thus patients usually present with acute tubular necrosis with oliguric conditions. About $10 \%$ of the AKI patients require dialysis, and in-hospital death occurs in around 5\%. Early diagnosis and management, including removal of the stings, antianaphylactic treatment, optimal hydration, and intensive dialytic support in the context of rapidly progressive renal failure and anuria, can reduce morbidity and mortality.

Local plants and chemicals are important environmental nephrotoxic resources in rural parts of Asia, causing AKI through mechanisms including direct injury to tubular cells, inducing renal ischemia, pigmenturia, or allergic acute interstitial nephritis. For example, AKI can be caused by taking certain species of mushrooms through direct cellular toxicity and indirect hypovolemia, djen- kolic acid crystals formed after ingestion of djenkol beans, and oxalate crystals formed after taking large amounts of star fruit juice or irumban puli juice.

\section{Obstetric AKI}

$\mathrm{AKI}$ is one of the most challenging complications of pregnancy in the tropical regions and is frequently related to suboptimal antenatal care, out-of-hospital delivery in rural areas, and unsafe abortions conducted by unqualified personnel or using insecure medicines [24]. In India, the improvements in prematernity medical care and obstetric care have decreased the incidence of obstetric AKI during the past 20 years, whereas the contribution of obstetric causes to all AKI cases is still 10-12\% [47]. In other Asian countries, such as Indonesia, Thailand, Pakistan, and Nepal, obstetric AKI constitutes up to $25-30 \%$ of all AKI entities [48, 49].

There are two peaks of AKI frequency during the course of gestation. The first peak, between 8 and 16 weeks of pregnancy, is mainly associated with septic abortion, nephrotoxicity from abortion medicines, and hyperemesis gravidarum. The second peak is during the period of the third trimester and past partum, and is related to preeclampsia, eclampsia, placental abruption, postpartum hemorrhage, disseminated intravascular coagulation, and puerperal sepsis. Most of the AKI cases (80\%) occur during the second peak [50]. Renal ischemia, caused by hemorrhagic shock or hypotension due to sepsis, is the dominant factor leading to AKI. Acute tubular necrosis is the most common clinical pattern, and acute cortical necrosis accounts for $22-25 \%$ of obstetric AKI [50]. Oliguria-anuria is seen in more than $85 \%$ of obstetric AKI patients, and about $90 \%$ of the cases require dialysis. The in-hospital death rate is $7-16 \%[50,51]$, fetal loss is about $50 \%$, and renal recovery at hospital discharge is around $50 \%$. In a recently published study from a tertiary hospital in India [50], only $40 \%$ of the obstetric AKI patients had received antenatal care, and $60 \%$ had hospital delivery. At hospital presentation, nearly $80 \%$ of the cases had sepsis, $1 / 3$ had abnormal consciousness, and $21 \%$ developed anuria. These numbers reveal a big burden from obstetric AKI in tropical Asian countries. Medical care for pregnant women is still deficient, and the timing of hospital referral in obstetric AKI is generally delayed. Extensive education of the communities as well as of the local medical care practitioners, setting up strict legalization and regulation of abortions, raising standards of living, and improving the overall obstetric medical care system in rural areas are the key steps to reduce obstetric AKI and promote outcomes. 
In China, the incidence of obstetric AKI is known to have decreased dramatically during the past 50 years, from $20-40 \%$ in the 1960 s to about $1 \%$ in recent years, primarily due to the legalization of abortion and improvements in antenatal care. However, most of the obstetric AKI cases ( $~ 80 \%)$ come from rural regions, and only half of them have received regular prenatal supervision [52]. Preeclampsia or eclampsia accounts for $43 \%$ of the cases, postpartum hemorrhage is seen in $14 \%$, sepsis in $13 \%$, thrombotic microangiopathy in $10 \%$, and acute fatty liver in $9 \%$. Dialysis is needed in $1 / 3$ of the cases, and the mortality rate varies from 0 to $15 \%$. Therefore, obstetric AKI is still a challenge in China, particularly in rural regions. Antenatal care needs to be further strengthened, and more attention should be paid to the monitoring and management of hypertension and potential kidney involvement.

\section{Natural Disaster-Related AKI}

Large parts of Asia are prone to natural disasters such as earthquakes, landslides, floods, typhoons, and tsunamis. AKI is a common severe comorbidity in each condition, especially in connection with earthquakes [53]. However, it is difficult to estimate the incidence of AKI after disasters because a large number of patients with AKI probably have been undiagnosed and underreported. In victims from earthquakes, crush syndrome, developed as a result of rhabdomyolysis after being trapped under rubble, is the second most common cause of death, followed by asphyxia [53]. The systemic manifestations include hypovolemic shock, acute oliguric kidney injury, hyperkalemia, metabolic acidosis, arrhythmias, cardiac and respiratory failure, infections, and psychological trauma. The pattern of AKI at the beginning is usually prerenal; however, if the victim has not received proper hydration, acute tubular necrosis develops. The mortality rate in dialyzed crush syndrome-related AKI was reported to be as high as $40 \%$ [54], whereas rates from more recent disasters, such as the China [55] and Iran [56] earthquakes, were lower (15-20\%). The first goal in treating crushed victims is to prevent crush syndrome, and early, massive, individualized fluid resuscitation is the most important and efficient method to accomplish this goal [57]. Besides crush syndrome, several other factors also contribute to the development of disaster-related AKI. Traumatic bleeding can lead to hypovolemic shock which results in prerenal AKI and subsequently acute tubular necrosis, nephrotoxic drugs may contribute to at least $1 / 3$ of the trauma-related AKI, and penetrating trauma to the urinary tract system can cause postrenal AKI. Therefore, prevention and treat- ment of AKI after disasters should include early in situ evaluation and intervention as well as rigorous monitoring and protection of the kidney throughout the injury and recovery course.

\section{Recognition and Treatment of AKI in Asia}

\section{Recognition}

Timely diagnosis of AKI is essential to set up interventional strategies to minimize further renal injury. In developed counties, it is reported that about $10 \%$ of AKI cases have not been recognized by the attending doctors [58], whereas the recently published national survey of AKI in China reveals a dramatic nonrecognition rate of AKI across the country [4]. About 3/4 of the AKI patients had not been recognized by the physicians in charge, and patients coming from less economically developed regions were at a higher risk for underrecognition of AKI. It is not surprising that delayed recognition of AKI is an independent risk factor for mortality [4]. In addition, as has been mentioned above, there are a large number of hospitalized patients who have not received sufficient serum creatinine tests $[4,5]$ and, therefore, cannot be defined as cases of AKI. A similar situation might also exist in other Asian developing countries where an inexplicably low prevalence of AKI has been reported [59]. This situation proves to be a big challenge, and there is an urgent need for health-care professionals to improve the recognition and diagnosis of AKI.

\section{Renal Referral}

It has been shown that delayed or absent renal referral is associated with a higher mortality rate, dialysis dependence, and length of hospital stay $[60,61]$. Based on the national survey from China, only $21 \%$ of the AKI cases had consulted a nephrologist, and renal referral is an independent protective factor against in-hospital death [4]. This underscores the need to form specialist renal teams to provide appropriate care for patients at risk of or with AKI. However, as has been pointed out by the ISN 0 by 25 initiative for AKI commission, the low-income developing countries have a very limited number of trained nephrologists and, thus, cannot provide sufficient professional care for the huge number of AKI cases. Therefore, training the entire health-care workers including the primary care physicians to raise the awareness, enable prompt identification of patients with suspected AKI, and provide practical management of $\mathrm{AKI}$ is of particular importance in low-income regions including Asian countries. 
Renal Replacement Therapy

Dialysis is available in most Asian urban centers; however, rural areas, especially remote areas up in the high mountains and inside forests, often lack the necessary resources. Peritoneal dialysis (PD) is an easy, less expensive, and effective renal replacement therapy (RRT) modality. Several studies have shown that PD, especially when high fluid volumes are used, has a similar effectiveness to extracorporeal blood purification in AKI patients $[62,63]$ and, thus, is a good option for RRT especially in low-income regions and pediatric AKI settings. PD has been used to successfully save lives in tropical Asian regions in various AKI settings such as malaria, snakebites, etc. However, in the national survey of AKI in China, nearly half of the patients that had RRT indications did not get the treatment, sometimes due to economic reasons; on the other hand, although resources for PD were available in all the study centers, only $1 \%$ of the dialyzed patients chose PD [4]. Therefore, medical resources are not the sole problem; doctors' acknowledgement and understanding of PD as a good option for AKI are important to promote the utility of $\mathrm{PD}$ in treating AKI cases.

\section{Future Perspectives on Improving the Management of AKI in Asia}

AKI in Asia has various epidemiological and clinical patterns in countries with different climates and socioeconomic features and, therefore, needs improved individualized approaches in these different regions. The cur- rent attention paid to AKI is clearly insufficient, and it is necessary to increase public awareness, provide clinical education and training, and form specialist renal teams to treat severe AKI patients. Most CA-AKI settings are potentially preventable. Prevention requires a broad-based public health approach, including improvement of living conditions such as providing safe water and food, efficient control of infection and vectors of disease, better obstetric care, vigorous surveillance of the pharmaceutical market, and raising awareness about the safe use of drugs, especially herbs, pesticides, and chemicals. Early fluid resuscitation, effective anti-infectious treatment, appropriate antidotes, and timely referral of patients with established AKI to nephrologists and to centers with dialysis facilities will alleviate AKI and improve outcomes. The choice of RRT treatment should fit patients' needs, and $\mathrm{PD}$ can be practiced more frequently in the treatment of AKI patients.

\section{Acknowledgements}

This work was supported by grants from the National Project 985 to Peking University for Clinical Study on Cooperation, the Beijing Training Program for Talents (20110009001000002), the National Natural Science Foundation of China (81270777), and the ISN Research Committee.

\section{Conflict of Interest Statement}

The author has no competing financial interests to declare.

\section{References}

1 International Society of Nephrology: Advancing Nephrology around the World. http:// www.theisn.org/initiatives/0x25-initiative? showall $=\&$ start $=1$.

-2 Mehta RL, Cerdá J, Burdmann EA, Tonelli M, Garcia-Garcia G, Jha V, Susantitaphong P, Rocco M, Vanholder R, Sever MS, Cruz D, Jaber B, Lameire $\mathrm{NH}$, Lombardi R, Lewington A, Feehally J, Finkelstein F, Levin N, Pannu N, Thomas B, Aronoff-Spencer E, Remuzzi G: International Society of Nephrology's 0 by 25 initiative for acute kidney injury (zero preventable deaths by 2025): a human rights case for nephrology. Lancet 2015;385:2616-2643.

- 3 Susantitaphong P, Cruz DN, Cerda J, et al: World incidence of AKI: a meta-analysis. Clin J Am Soc Nephrol 2013;8:1482-1493.

-4 Yang L, Xing G, Wang L, et al: Acute kidney injury in China: a cross-sectional survey. Lancet 2015;386:1465-1471.
5 Xu X, Nie S, Liu Z, et al: Epidemiology and clinical correlates of AKI in Chinese hospitalized adults. Clin J Am Soc Nephrol 2015;10: 1510-1518.

6 Fujii T, Uchino S, Takinami M, et al: Subacute kidney injury in hospitalized patients. Clin J Am Soc Nephrol 2014;9:457-461.

$\checkmark 7$ Kwon SH, Noh H, Jeon JS, et al: An assessment of AKIN criteria for hospital-acquired acute kidney injury: a prospective observational cohort study. Nephron Clin Pract 2010; 116:c217-c223.

-8 Ulusoy S, Arı D, Ozkan G, et al: The frequency and outcome of acute kidney injury in a tertiary hospital: which factors affect mortality? Artif Organs 2015;39:597-606.

-9 Uchino S, Bellomo R, Goldsmith D, et al: An assessment of the RIFLE criteria for acute renal failure in hospitalized patients. Crit Care Med 2006;34:1913-1917.
10 Thomas M, Sitch A, Dowswell G: The initial development and assessment of an automatic alert warning of acute kidney injury. Nephrol Dial Transplant 2011;26:2161-2168.

11 Srisawat N, Kellum JA: Acute kidney injury: definition, epidemiology, and outcome. Curr Opin Crit Care 2011;17:548-555.

12 Zeng X, McMahon GM, Brunelli SM, et al: Incidence, outcomes, and comparisons across definitions of AKI in hospitalized individuals. Clin J Am Soc Nephrol 2014;9:12-20.

13 Shema L, Ore L, Geron R, et al: Hospital-acquired acute kidney injury in Israel. Isr Med Assoc J 2009;11:269-274.

14 Xuying L, Li J, Bin D, Ying W, et al: A comparison of different diagnostic criteria of acute kidney injury in critically ill patients. Crit Care 2014;18:R144. 
15 Wen Y, Jiang L, Xu Y, et al: Prevalence, risk factors, clinical course, and outcome of acute kidney injury in Chinese intensive care units: a prospective cohort study. Chin Med J (Engl) 2013;126:4409-4416.

16 Park WY, Hwang EA, Jang MH, et al: The risk factors and outcome of acute kidney injury in the intensive care units. Korean J Intern Med 2010;25:181-187.

17 Shinjo H, Sato W, Imai E, et al: Comparison of kidney disease: improving global outcomes and acute kidney injury network criteria for assessing patients in intensive care units. Clin Exp Nephrol 2014;18:737-745.

18 Wijewickrama ES, Ratnayake GM, Wikramaratne $\mathrm{C}$, et al: Incidences and clinical outcomes of acute kidney injury in ICU: a prospective observational study in Sri Lanka. BMC Res Notes 2014;7:305-312.

$\checkmark 19$ Kohli HS, Bhat A, Jairam A, et al: Predictors of mortality in acute renal failure in a developing country: a prospective study. Ren Fail 2007;29:463-469.

20 Kumar S, Raina S, Vikrant S, et al: Spectrum of acute kidney injury in the Himalayan region. Indian J Nephrol 2012;22:363-366.

21 Prakash J, Singh TB, Ghosh B, et al: Changing epidemiology of community-acquired acute kidney injury in developing countries: analysis of 2,405 cases in 26 years from eastern India. Clin Kidney J 2013;6:150-155.

22 Mehta RL, Pascual MT, Soroko S, et al: Program to Improve Care in Acute Renal Disease Spectrum of acute renal failure in the intensive care unit: the PICARD experience. Kidney Int 2004;66:1613-1621.

-23 Uchino S, Kellum JA, Bellomo R, et al; Beginning and Ending Supportive Therapy for the Kidney (BEST Kidney) Investigators: Acute renal failure in critically ill patients: a multinational, multicenter study. JAMA 2005;294 813-818.

24 Jha V, Chugh KS: Community-acquired acute kidney injury in Asia. Semin Nephrol 2008; 28:330-347.

-25 Naqvi R, Ahmad E, Akhtar F, et al: Outcome in severe acute renal failure associated with malaria. Nephrol Dial Transplant 2003;18: 1820-1823.

26 Saravu K, Rishikesh K, Parikh CR: Risk factors and outcomes stratified by severity of acute kidney injury in malaria. PLoS One 2014;9:e90419.

27 Mishra SK, Das BS: Malaria and acute kidney injury. Semin Nephrol 2008;28:395-408

28 Barsoum RS: Malarial acute renal failure. J Am Soc Nephrol 2000;11:2147-2154.

29 Plewes K, Haider MS, Kingston HW, et al: Severe falciparum malaria treated with artesunate complicated by delayed onset haemolysis and acute kidney injury. Malar J 2015;14:246-254.

30 Erdinc FS, Koruk ST, Hatipoglu CA, et al: Three cases of anicteric leptospirosis from Turkey: mild to severe complications. J Infect 2006;52:e45-e48.

-31 Cengiz K, Sahan C, Sunbul M, et al: Acute renal failure in leptospirosis in the black-sea re- gion in Turkey. Int Urol Nephrol 2002;33: 133-136.

32 Herath NJ, Kularatne SA, Weerakoon KG, et al: Long term outcome of acute kidney injury due to leptospirosis? A longitudinal study in Sri Lanka. BMC Res Notes 2014;7:398.

33 Goswami RP, Goswami RP, Basu A, et al: Predictors of mortality in leptospirosis: an observational study from two hospitals in Kolkata, eastern India. Trans R Soc Trop Med Hyg 2014;108:791-796.

34 Datta S, Sarkar RN, Biswas A, et al: Leptospirosis: an institutional experience. J Indian Med Assoc 2011;109:737-738

35 Xiao H, Lin X, Gao L, et al: Ecology and geography of hemorrhagic fever with renal syndrome in Changsha, China. BMC Infect Dis 2013;13:305.

-36 Liu X, Jiang B, Bi P, et al: Prevalence of haemorrhagic fever with renal syndrome in mainland China: analysis of National Surveillance Data, 2004-2009. Epidemiol Infect 2012;140: 851-857.

37 Han D, Liu Z, Han Q, et al: Acute kidney injury in patients with hemorrhagic fever with renal syndrome caused by Hantaan virus: comparative evaluation by RIFLE and AKIN criteria. Vector Borne Zoonotic Dis 2011;11: 723-730.

38 Outinen TK, Mäkelä S, Clement J, et al: Community acquired severe acute kidney injury caused by hantavirus-induced hemorrhagic fever with renal syndrome has a favorable outcome. Nephron 2015;130:182-190.

39 Gnemmi V, Verine J, Vrigneaud L, et al: Microvascular inflammation and acute tubular necrosis are major histologic features of hantavirus nephropathy. Hum Pathol 2015; 46:827-835.

40 Prakash J, Tripathi K, Malhotra V, et al: Acute renal failure in eastern India. Nephrol Dial Transplant 1995; 10:2009-2012.

41 Pinho FMO, Zanetta DMT, Burdmann EA: Acute renal failure after Crotalus durissus snakebite. A prospective survey on $100 \mathrm{pa}$ tients. Kidney Int 2005;67:659-667.

42 Kanjanabuch T, Sitprija V: Snakebite nephrotoxicity in Asia. Semin Nephrol 2008;28:363372.

43 Xie C, Xu S, Ding F, et al: Clinical features of severe wasp sting patients with dominantly toxic reaction: analysis of 1,091 cases. PLoS One 2013;8:e83164.

44 Zhang L, Yang Y, Tang Y, et al: Recovery from AKI following multiple wasp stings: a case series. Clin J Am Soc Nephrol 2013;8:18501856.

45 Sigdel MR, Raut KB: Wasp bite in a referral hospital in Nepal. J Nepal Health Res Counc 2013;11:244-250.

46 Xuan BH, Mai HL, Thi TX, et al: Swarming hornet attacks: shock and acute kidney injury - a large case series from Vietnam. Nephrol Dial Transplant 2010;25:1146-1150.

47 Godara SM, Kute VB, Trivedi HL, et al: Clinical profile and outcome of acute kidney injury related to pregnancy in developing coun- tries: a single-center study from India. Saudi J Kidney Dis Transpl 2014;25:906-911.

48 Rana A, Pradhan N, Gurung G, et al: Induced septic abortion: a major factor in maternal mortality and morbidity. J Obstet Gynaecol Res 2004;30:3-8.

49 Khakurel S, Satyal PR, Agrawal RK, et al: Acute renal failure in a tertiary care center in Nepal. J Nephrol Med Assoc 2005;44:32-35.

50 Godara SM, Kute VB, Trivedi HL: Clinical profile and outcome of acute kidney injury related to pregnancy in developing countries: a single-center study from India. Saudi J Kidney Dis Transpl 2014;25:906-911.

51 Ali A, Ali MA, Ali MU, et al: Hospital outcomes of obstetrical-related acute renal failure in a tertiary care teaching hospital. Ren Fail 2011;33:285-290.

52 Liu YM, Bao HD, Jiang ZZ, et al: Pregnancyrelated acute kidney injury and a review of the literature in China. Intern Med 2015;54: 1695-1703.

-53 Sever MS, Lameire N, Van Biesen W, Vanholder R: Disaster nephrology: a new concept for an old problem. Clin Kidney J 2015;8:300-309.

54 Oda J, Tanaka H, Yoshioka T, et al: Analysis of 372 patients with crush syndrome caused by the Hanshin-Awaji earthquake. J Trauma 1997;42:470-475.

$55 \mathrm{He}$ Q, Wang F, Li G, et al: Crush syndrome and acute kidney injury in the Wenchuan Earthquake. J Trauma 2011;70:1213-1217.

56 Hatamizadeh P, Najafi I, Vanholder R, et al: Epidemiologic aspects of the Bam earthquake in Iran: the nephrologic perspective. Am J Kidney Dis 2006;47:428-438.

57 Sever MS, Vanholder R: Recommendations for the management of crush victims in mass disasters. Nephrol Dial Transplant 2012; 27(suppl 1):i1-i67.

58 Meran S, Wonnacott A, Amphlett B, et al: How good are we at managing acute kidney injury in hospital? Clin Kidney J 2014;7:144150

59 Singh TB, Rathore SS, Choudhury TA, et al: Hospital-acquired acute kidney injury in medical, surgical, and intensive care unit: a comparative study. Indian J Nephrol 2013;23: 24-29.

60 Costa e Silva VT, Liano F, Muriel A, et al: Nephrology referral and outcomes in critically ill acute kidney injury patients. PLoS One 2013; 8:e70482.

61 Ponce D, Zorzenon CP, dos Santos NY, et al: Early nephrology consultation can have an impact on outcome of acute kidney injury patients. Nephrol Dial Transplant 2011;26: 3202-3206.

62 Ponce D, Berbel MN, Abrao JM, et al: A randomized clinical trial of high volume peritoneal dialysis versus extended daily hemodialysis for acute kidney injury patients. Int Urol Nephrol 2013;45:869-878.

63 Bonilla-Felix M: Peritoneal dialysis in the pediatric intensive care unit setting: techniques, quantitations and outcomes. Blood Purif 2013;35:77-80. 